\title{
Homebase: Successfully using the principles of assertive community treatment to decrease emergency department and hospital utilization among super-utilizers
}

Natasha T. Cunningham ${ }^{1,2}$, Mark Sendak ${ }^{3}$, Marigny Bratcher ${ }^{4}, J_{u l i a}$ Gamble $^{5}$, Janice Dillard $^{6}$, Lynn Bowlby $^{5}$, Lawrence Greenblatt ${ }^{5}$, Daniella A. Zipkin ${ }^{5}$ and Alex Cho ${ }^{5}$

${ }^{1}$ Duke University Department of Psychiatry, Division of Social and Community Psychiatry, USA

${ }^{2}$ Kaiser Permanente, Southern California Permanente Group, USA

${ }^{3}$ Duke Institute for Health Innovation, USA

${ }^{4}$ Duke University Department of Community and Family Medicine, Division of Community Health, USA

${ }^{5}$ Duke University Department of Internal Medicine, Division of General Internal Medicine, USA

${ }^{6}$ Duke University Department of Case Management, Division of Social Work, USA

\begin{abstract}
In the 1960s, deinstitutionalization drove psychiatric care away from the inpatient setting and increased the emphasis on outpatient care. In this climate, Assertive Community Treatment Teams (ACTT) became a widespread strategy to manage psychiatrically complex patients in the community. Since the 2010s, the creation of the Affordable Care Act has led to a type of medical deinstitutionalization with an increased focus on managing medically complex patients- "super-utilizers"-in the outpatient setting. Using the principles of ACTT, we created Homebase: a multidisciplinary, patient centered, care manager driven intervention focusing on identifying and addressing barriers to medical care among super-utilizers and promoting engagement with their medical home. This intervention resulted in a $43 \%$ reduction in both $\mathrm{ED}$ visits and hospital days and $\$ 839,892$ direct cost savings over the first year. When designing programs to optimize care for super-utilizers, the principles of ACTT are readily transferable from psychiatrically complex to medically complex populations.
\end{abstract}

\begin{abstract}
Abbreviations: ACTT: Assertive Community Treatment Teams; ED: Emergency Department; ACO: Accountable Care Organization; DOC: Duke Outpatient Clinic; COPD: Chronic Obstructive Pulmonary Disease.
\end{abstract}

\section{Introduction}

In the 1960s, psychiatry underwent the process of deinstitutionalization where a significant and rapid decrease in the number of psychiatric hospital beds led to a push to support patients with severe persistent mental illness living in the community [1]. In 1980, Stein and Tess reported on a program providing support for this patient population in Madison, Wisconsin. This program, originally named Training in Community Living, was expanded and developed into what are currently known as Assertive Community Treatment Teams (ACTT) [2-4]. Mueser summarizes the findings of Surles and McGurrin by stating that ACTT "emerged out of a growing recognition that many patients with severe psychiatric disorders could not be engaged in treatment using traditional case management practices, yet they consumed many of the most costly treatment services, such as emergency room visits. [5]" ACTT experienced rapid uptake in the years after its introduction, likely due to the significant decrease in hospitalizations seen in early studies. Specifically, Tom Burns notes an increase from 8 trials in a 1981 review $^{6}$ to 75 trials in a 1998 review [6].

The creation of the Affordable Care Act of 2010 has led to the creation of Accountable Care Organizations (ACOs). ACOs are eligible for "shared savings" reimbursement for providing quality care at a relatively lower cost than previous years. Thus, health systems rather than insurance companies, shoulder some of the financial risk associated with high utilization of medical resources [7]. This structural change has led to a type of medical deinstitutionalization with direction of care away from the inpatient setting and towards the more cost effective patient centered medical home.

In this climate, healthcare systems have become invested in targeting high utilizers of emergency and inpatient care, called "superutilizers". Several programs have risen throughout the country using care management to decrease the need for Emergency Department (ED) and inpatient care among these individuals [8]. Parallels with the Assertive Community Treatment Teams of the 1990s include a goal of supporting patients in the outpatient setting and the use of care management as a primary tool. Here we describe our Homebase program: a novel, care manager driven intervention targeting superutilizers with an explicit foundation in the principles of Assertive

Correspondence to: Natasha Cunningham, MD, 1900 East 4th St, Santa Ana, CA 92704, USA, Tel: 603-401-0593; Fax: 714-967-4575; E-mail: Natasha.cunningham@duke.edu

Key words: super-utilizers, primary care redesign, patient centered care, community health, mental health, access to care, case management

Received: August 18, 2016; Accepted: September 02, 2016; Published: September 07, 2016 
Community Treatment. Specifically, use of the biopsychosocial model of understanding patient health, multidisciplinary care teams, community outreach and the support of recovery rather than dependence [9]. This intervention demonstrates that the lessons learned from psychiatric deinstitutionalization and the development of ACTT can be successfully applied to projects targeting medical superutilizers in the current climate of medical deinstitutionalization.

\section{Methods}

\section{Population}

The Duke Outpatient (DOC) clinic provides primary care for approximately 4500 adults in Durham County. Patients are attributed to the DOC as their medical home if they have completed 2 appointments at the clinic within the past 36 months and at least 1 appointment within the past 12 months. Inclusion criteria for the Homebase intervention were 6 or more ED visits or hospitalizations in the past 6 months with a pattern of ongoing ED utilization. Patients were identified through North Carolina Medicaid and Duke Hospital data quantifying ED use for all DOC patients and through direct referral by providers.

\section{Intervention}

The Homebase team consists of a full time RN care manager, an MD dually trained in internal medicine and psychiatry, an NP with extensive knowledge of community resources, and an LCSW who had been located at the clinic for many years. After patients are identified for enrollment, the care manager performs a retrospective chart review identifying patterns of utilization and potential areas for intervention. The team then develops an individual care plan and places it in the patient's electronic medical record. The care manager proactively reaches out to the patient to cooperatively address barriers to medical care and drivers of ED utilization. Interaction with the patient is broken down into two 6 month phases. First, an "engagement" phase when patient driven goals are identified and addressed and focus is given to improved relationship and connection with clinic medical providers. This is followed by a 6 month "action" phase during which explicit focus is placed on improved medical health and decreased ED dependence. The team receives real-time electronic notification when enrolled patients arrive in the ED and can coordinate care with ED providers. Enrolled patients are provided enhanced walk-in access to the clinic, and are frequently co-managed by a resident primary care provider and the nurse practitioner. The team holds twice weekly meetings to discuss new patients and those with acute needs or increased utilization. Patients are reviewed every 6 months following enrollment to assess response to the intervention and the effectiveness of engagement strategies.

\section{Outcomes}

The primary outcome is the change in the number of ED visits and inpatient days for enrolled patients in the year before compared to the year after enrollment in the Homebase program. The secondary outcome is monetary savings to the healthcare system, which was calculated using the average direct cost of an ED visit (\$497) and an inpatient day (\$2000) for DOC patients during the prior fiscal year. All data was pulled from the Duke University Health System data warehouse and analysis was completed using $\mathrm{R}$ version 3.0.2.

This project was granted exemption by the Duke University Institutional Review Board.

\section{Results}

Eighty-seven patients were enrolled in the intervention within the first 2 years. Of those, 25 had been enrolled for 12 months or more and were included in the analysis. Of the analyzed patients, $76 \%$ had documented mental health (MH) diagnoses and 52\% had documented substance abuse (SA) disorders. Other common diagnoses among this population included diabetes, chronic obstructive pulmonary disease (COPD)/asthma and chronic kidney disease (CKD). The majority of enrolled patients were insured through Medicare, Medicaid or both. Forty-four percent of analyzed patients struggled with housing instability and difficulty affording medications, and over $60 \%$ had problems with transportation to clinic appointments (Table 1).

Among the 25 patients included in the analysis, a 43\% reduction was seen in both ED visits and hospital days over the first year of enrollment in the Homebase program compared to the previous year (Figure 1). The average number of ED visits per patient decreased from 14 per year to 8 per year and the average number of hospital days decreased from 35 to 20 per year. The decrease in hospital days was accounted for by both a $28 \%$ reduction in the number of hospitalizations (from

Table 1. Homebase patient demographics $(\mathrm{N}=25)$.

\begin{tabular}{|c|c|}
\hline Age (Mean, range) & 45.8 years $(24-78$ years $)$ \\
\hline \multicolumn{2}{|l|}{ Diagnoses; $(\mathrm{N}, \%)$} \\
\hline \begin{tabular}{|l|l} 
Mental illness \\
\end{tabular} & $19(76)$ \\
\hline Substance abuse & $13(52)$ \\
\hline Diabetes & $15(60)$ \\
\hline Congestive Heart Failure & $7(28)$ \\
\hline COPD/Asthma & $17(68)$ \\
\hline CKD & $10(40)$ \\
\hline \multicolumn{2}{|l|}{ Insurance $(\mathrm{N}, \%)$} \\
\hline Medicaid & $14(56)$ \\
\hline Medicare & $13(52)$ \\
\hline Uninsured & $1(4)$ \\
\hline Private Insurance & $7(28)$ \\
\hline \multicolumn{2}{|l|}{ Identified social barriers (N, \%) } \\
\hline \begin{tabular}{|l|l} 
Housing instability
\end{tabular} & $11(44)$ \\
\hline Food insecurity & $4(16)$ \\
\hline Difficulty affording medications & $11(44)$ \\
\hline Inadequate transportation & $16(64)$ \\
\hline
\end{tabular}

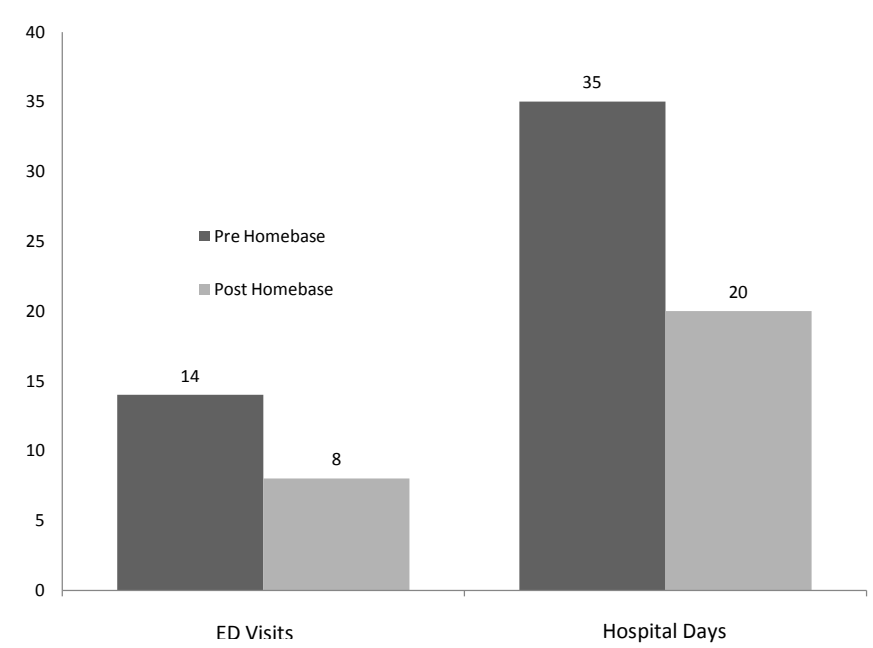

Figure 1. Average ED visits and hospital days per patient in the 12 months before and 12 months after Homebase enrollment. 


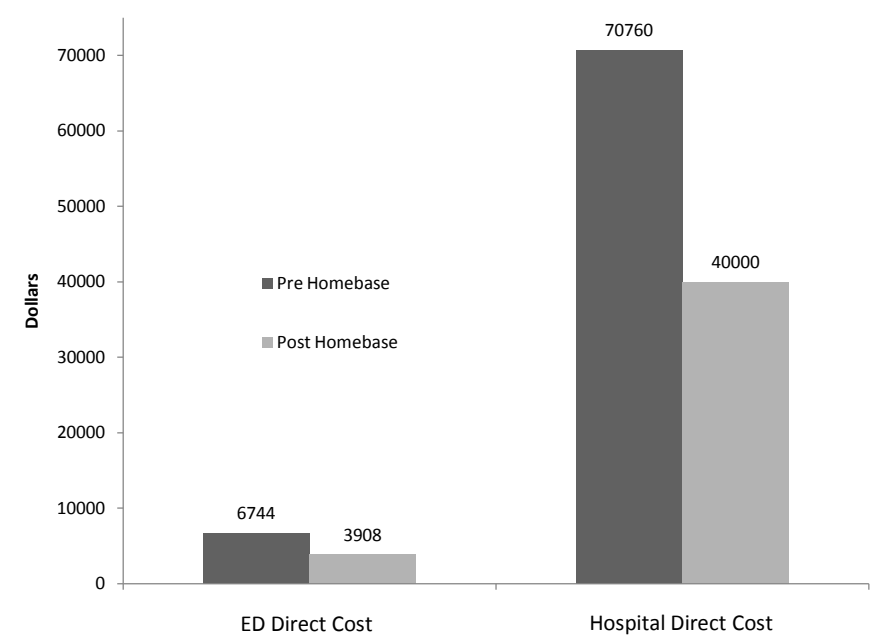

Figure 2. Average direct ED and admission cost per patient 12 months before and 12 months after Homebase enrollment.

2.84 per year to 2.04 per year) and a $21 \%$ reduction in length of stay per hospitalization (from 12.5 days per admission to 9.8 days per admission). This equated to a direct cost savings of $\$ 2,837$ per patient in $\mathrm{ED}$ costs and $\$ 30,760$ per patient in admission costs. This resulted in a total savings of $\$ 839,892$ to the health system over the course of 1 year for the first 25 patients enrolled (Figure 2).

\section{Discussion}

This evaluation shows that the principles of ACTT can be effectively adapted to support super-utilizers of medical care in redirecting their care towards outpatient resources. The lessons learned from psychiatric deinstitutionalization in the 1990s can be applied to the current climate of medical deinstitutionalization. The use of a biopsychosocial model is central to the patient centered medical home along with the basis for ACTT and the Homebase intervention.

Although the patients targeted for this intervention had a relatively high rate of psychiatric comorbidity (MH 76\%, SA 52\%), this was only slightly higher than the clinic population as a whole (MH 50\%). By identifying high rates of ED utilization as a marker of psychosocial issues, we were able to target complex issues that are not easily captured through routine medical or psychiatric assessment and diagnosis.

Another strength of the Homebase intervention is the explicit focus on patient engagement. Identifying and initially targeting patient identified goals is consistent with the principles of illness management and recovery, which focus on targeting social and functional consequences of uncontrolled symptoms (not solely symptoms) to align providers and patients in the pursuit of both subjective and objective wellness [10]. Through proactive outreach the team was able to form relationships with patients and engage them with the clinic for acute and chronic medical needs. Over time, patients were willing to engage more fully in health promoting behavioral change leading to decreased need for urgent or emergent medical care.

Weaknesses of this analysis include the descriptive nature and the lack of a control group, since Hombase was initially created as a quality improvement initiative. Several randomized controlled trials (RCTs) exist showing the superiority of ACTT in decreasing psychiatric hospitalization and ED visits [1]. There are far fewer RCTs evaluating the use of care managers to target high utilizers of medical care. This is an important area for future research. As the interest in improving care for super-utilizers continues to increase, identifying effective models to shift patients' pattern of utilization towards their medical home will become a priority. This evaluation shows that the knowledge gained through psychiatric care management can be a useful foundation for programs targeting medical utilization.

The use of ACTT based principles to target medical super-utilizers emphasizes the artificial nature of the silos between medical and psychiatric care. Many providers are tempted to identify Homebase as a "medical ACTT team". In reality, the element that identifies ACTT as psychiatric and Homebase as medical is simply the diagnoses documented during ED visits and admissions. Both interventions use a biopsychosocial model to understand patients as multidimensional individuals and identify many areas of need. This is an essential cornerstone in the super-utilizer interventions throughout the country [8]. When developing interventions to support patient wellness in the community and emphasize care outside of a hospital setting, health systems and innovation leaders should turn to the principles used successfully in decades of ACTT care. This level of integration promotes an understanding of the multidimensional complexity of many superutilizers and is one step towards creating a health system that promotes whole person wellness rather than reacts to illness.

\section{Acknowledgements}

The authors would like to acknowledge the work of the Duke Outpatient Clinic Redesign Group and the support from Duke University Hospital and Northern Piedmont Community Care, which made the creation of Homebase possible. Homebase team members received funding from Duke University Hospital and Northern Piedmont Community Care. The authors report no proprietary or commercial interest in any product or concept discussed in this article.

\section{References}

1. Mueser KT, Bond GR, Drake RE, Resnick SG (1998) Models of community care for severe mental illness: a review of research on case management. Schizophr Bull 24: 37-74. [Crossref]

2. Test MA, Stein LI (1980) Alternative to mental hospital treatment. III. Social cost. Arch Gen Psychiatry 37: 409-412. [Crossref]

3. Stein LI, Test MA (1980). Alternative to mental hospital treatment. I. Conceptual model, treatment program, and clinical evaluation. Archives Of General Psychiatry 37: 392-397.

4. Weisbrod BA, Test MA, Stein LI (1980) Alternative to mental hospital treatment. II. Economic benefit-cost analysis. Arch Gen Psychiatry 37: 400-405. [Crossref]

5. Surles RC, McGurrin MC (1987). Increased use of psychiatric emergency services by young chronic mentally ill patients. Hospital \& Community Psychiatry 38: 401-405.

6. Braun P, Kochansky G, Shapiro R, Greenberg S, Gudeman JE, et al. (1981) Overview: deinstitutionalization of psychiatric patients, a critical review of outcome studies. Am J Psychiatry 138: 736-749. [Crossref]

7. Edwards ST, Abrams MK, Baron RJ, Berenson RA, Rich EC, et al. (2014) Structuring payment to medical homes after the affordable care act. J Gen Intern Med 29: 14101413. [Crossref]

8. Better Care for Super-Utilizers 2014

9. Burns $\mathrm{T}$ (2010) The rise and fall of assertive community treatment? Int Rev Psychiatry 22: 130-137. [Crossref]

10. Mueser KT, Meyer PS, Penn DL, Clancy R, Clancy DM, et al. (2006) The Illness Management and Recovery program: rationale, development, and preliminary findings. Schizophrenia bulletin. 32 Supp: S32-43.

Copyright: (C)2016 Cunningham NT. This is an open-access article distributed under the terms of the Creative Commons Attribution License, which permits unrestricted use, distribution, and reproduction in any medium, provided the original author and source are credited. 